\title{
Percutaneous and open cannulation for peripheral cardiopulmonary bypass: pearls and pitfalls
}

\author{
Alexander P. Nissen ${ }^{1,2}$, Juan Abreu ${ }^{1}$, Vahram Ornekian ${ }^{1}$, Stephanie Nguyen ${ }^{1}$, Tom C. Nguyen ${ }^{1}$ \\ ${ }^{1}$ Department of Cardiothoracic and Vascular Surgery, University of Texas Health Science Center Houston, McGovern Medical School, Houston, \\ TX, USA; ${ }^{2}$ Department of Surgery, San Antonio Military Medical Center, Fort Sam Houston, TX, USA \\ Correspondence to: Tom C. Nguyen, MD, FACS, FACC. Associate Professor Cardiothoracic Surgery, Director of Minimally Invasive Valve Surgery, \\ 6400 Fannin St, Suite 2850, Houston, TX 77030, USA. Email: tom.c.nguyen@gmail.com.
}

\begin{abstract}
Minimally invasive mitral surgery requires unique considerations for safe conduct of cardiopulmonary bypass. We describe several techniques, highlighting both percutaneous and direct femoral cannulation, with accompanying video illustrations, while addressing relevant pearls and pitfalls of each.
\end{abstract}

Keywords: Minimally invasive; femoral cannulation; cardiopulmonary bypass

Received: 11 September 2018; Accepted: 13 December 2018; Published: 30 December 2018.

doi: 10.21037 /jovs.2018.12.10

View this article at: http://dx.doi.org/10.21037/jovs.2018.12.10

\section{Introduction}

Minimally invasive techniques for mitral surgery were first reported in the late 1990s (1-4), and their utilization has continued to increase (5). Minimally invasive valve surgery requires unique considerations for cannulation, regardless of surgeon choice between right minithoracotomy with direct vision, endoscopic right minithoracotomy, or roboticassisted right minithoracotomy for surgical approach.

In this article, we sought to review various techniques for percutaneous and open cannulation techniques for peripheral cardiopulmonary bypass. The senior author's technique will be highlighted and illustrated through an accompanying video, with discussion of helpful methods to avoid common pitfalls. Additional approaches for cannulation, aortic occlusion, and myocardial protection will also be discussed.

\section{Preoperative preparation \& operative techniques}

\section{History and examination}

Determining candidacy for minimally invasive mitral surgery and appropriate cannulation begins with a thorough history and physical exam. Femoral cannulation is the most commonly utilized modality in our practice, and relevant considerations include the presence or absence of palpable femoral pulses, evidence of any unrepaired femoral or inguinal hernia, body habitus including presence of a large pannus, or active cutaneous fungal infection of either groin.

\section{Preoperative imaging}

CT angiography (CTA) of the chest, abdomen, and pelvis is strongly encouraged in the preoperative evaluation of patients who are candidates for minimally invasive mitral surgery, and a great deal of information can be garnered based on certain anatomic findings (6). Beginning with the chest, assessment for any aortic atheroma should include the degree of atheromatous burden, specific location, and the presence of hard or soft plaque. Significant atheromatous burden may preclude safe placement of either an aortic cross clamp or use of an endoaortic balloon for occlusion. Mid-ascending aortic size greater than $4 \mathrm{~cm}$ may also limit complete aortic occlusion when using the endoaortic balloon (7). Preoperative planning for peripheral cannulation requires thorough assessment of the descending aorta and iliofemoral vasculature as well. Significant atheromatous disease may preclude safe femoral cannulation and retrograde perfusion, due to increased risk of stroke. Iliofemoral calcification, aneurysmal disease, or dissection should also be ruled out to ensure safe vascular access for peripheral cannulation. The presence of an inferior vena 
Table 1 Relevant preoperative CTA findings for peripheral cannulation and conduct of CPB

\begin{tabular}{ll}
\hline Location & Findings \\
\hline Ascending aorta & Significant atheromatous burden precluding safe clamp placement or endoaortic balloon occlusion \\
& Size $>4 \mathrm{~cm}$ associated with incomplete balloon occlusion \\
Great vessels & Aberrant anatomy including persistent left SVC, aberrant right subclavian artery, or patent ductus arteriosus \\
Descending aorta & $\begin{array}{l}\text { Significant atheroma, or AAA with mural thrombus, associated with increased risk of stroke during retrograde } \\
\text { perfusion }\end{array}$ \\
Iliofemoral & Presence of significant plaque, aneurysm, tortuosity, or dissection limiting safe arterial canula access \\
Venous anatomy & Presence of an IVC filter \\
& Presence of persistent left sided SVC \\
\hline
\end{tabular}

CTA, CT angiography; CPB, cardiopulmonary bypass; IVC, inferior vena cava; SVC, superior vena cava; AAA, abdominal aortic aneurysm.

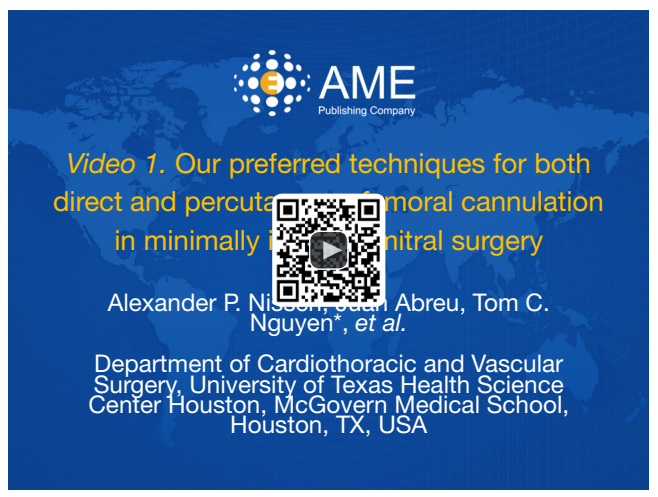

Figure 1 Our preferred techniques for both direct and percutaneous femoral cannulation in minimally invasive mitral surgery (8). Available online: http://www.asvide.com/article/view/29187

cava (IVC) filter could also exclude safe venous cannulation. We have listed what we consider relevant CTA findings as they pertain to peripheral cannulation for cardiopulmonary bypass (CPB) in Table 1 .

Preoperative echocardiography, including a high quality transthoracic (TTE) or transesophageal (TEE), may also demonstrate significant aortic atheroma, or an enlarged coronary sinus and associated persistent left-sided superior vena cava (SVC), in conjunction with the above-described CTA findings.

\section{Direct approaches}

\section{Venous cannulation}

\section{Femoral venous access}

Figure 1 demonstrates our standard techniques for peripheral cannulation (open and percutaneous) in minimally invasive mitral surgery. The site of both femoral arteries is marked prior to incision. A $2-3 \mathrm{~cm}$ incision is made below the inguinal ligament, and dissection carried down until the anterior aspect of both the common femoral artery (CFA) and vein are exposed. Exposure of the inguinal ligament is a helpful landmark to ensure cannulation of the common femoral vessels, and avoid cannulation of either the superficial or profunda branches. Use of the inguinal ligament as an anatomic landmark can be especially helpful in obese patients. Avoid excessive dissection, especially medially, to prevent lymphatic leak. A purse-string suture is placed on the anterior aspect of the common femoral vein with 4-0 polypropylene. The patient is fully heparinized prior to cannulation. We generally use a $25 \mathrm{Fr}$ venous canula for most patients. Prior to cannulation, we routinely measure the approximate site of insertion by placing the canula on the patient. Using the Seldinger technique, wire access is obtained to the common femoral vein, and advanced into the SVC, with TEE confirmation by a bicaval view. Wire access to the SVC, and not the right atrium or ventricle, is key prior to canula insertion to prevent right atrial or ventricular perforation with canula advancement. After guidewire access, the canula may be advanced with placement of the distal tip in the SVC, and secured in place. We routinely use vacuum-assisted venous drainage, though kinetic-assisted drainage may also prove adequate. At case conclusion the purse-string is used for venotomy repair.

\section{Troubleshooting venous access}

If despite several attempts at gentle wire advancement, the operator cannot obtain SVC access, there are several options to facilitate safe placement. One is to ask the anesthesia team to perform a Valsalva maneuver, which 
will increase right atrial pressure and may aid in SVC, as opposed to right atrial, wire access. Another option is to place a slight bend in the wire tip. It may also be required to use fluoroscopy guidance (e.g., use of a mobile C-arm device) to ensure adequate SVC wire access, if TEE visualization is difficult. Lastly, another approach could involve advancing a bent multipurpose catheter and directing the catheter towards the SVC under ECHO guidance followed by advancing a soft-tip J-wire.

If after safe guidewire access, the venous canula meets resistance, ensure the venotomy has been adequately dilated. If it has not, the venotomy may be extended slightly with a number 11 blade to reduce resistance at the site of insertion. If adequate bicaval drainage cannot be obtained via femoral access alone, SVC drainage may be augmented by internal jugular vein cannulation by the anesthesia team.

\section{Arterial cannulation}

\section{Direct femoral cannulation}

After femoral venous cannulation, attention is turned to the CFA. A 4-0 polypropylene purse-string suture is placed on the anterior aspect of the artery. The artery is similarly accessed using the Seldinger technique. We recommend the approach of choosing the smallest arterial canula size that will provide adequate perfusion, in order to mitigate the risk of limb ischemia, as increasing canula size has been shown to be an independent risk factor for limb ischemia in minimally invasive cardiac surgery (9). Our general approach when determining appropriate canula size uses body surface area (BSA) as follows: 15 Fr canula for BSA $\leq 1.6 \mathrm{~m}^{2}, 17 \mathrm{Fr}$ canula for BSA $1.7-2.1 \mathrm{~m}^{2}$, and $19 \mathrm{Fr}$ canula for $\mathrm{BSA} \geq 2.1 \mathrm{~m}^{2}$. Meticulous technique and gentle advancement of all wires and canulas is critical to avoid access vessel injury. TEE visualization of the descending aorta during arterial cannulation and initiation of CPB is used to ensure the rare event of retrograde dissection has not occurred. At case conclusion, the previously placed purse-string suture is used for arteriotomy closure, taking care to avoid stenosis. The presence of a distal pulse is confirmed, and the groin incision is closed in standard fashion.

\section{Troubleshooting direct femoral access}

Occasionally, significant iliofemoral tortuosity will prevent safe advancement of the arterial inflow cannula for CPB. In this case, after safe guidewire access to the descending aorta confirmed by TEE, a glide catheter may be advanced over the originally placed soft guidewire. This allows wire exchange for a stiff guidewire to straighten the arterial segment in question. Hereafter, the glide catheter may be removed and arterial cannulation for $\mathrm{CPB}$ attempted over the stiff guidewire.

Similar to venous cannulation, ensuring adequate arteriotomy dilation prior to cannulation is key to minimize unnecessary pressure during insertion. If dilation is inadequate, extending the arteriotomy slightly with a number 11 blade can assist in safe arterial canula advancement.

\section{Femoral side-arm graft technique}

For patients with narrow iliofemoral access lacking significant calcification, surgeons may choose to cannulate a side-arm graft for arterial access. After femoral exposure and full heparinization as described above, vascular clamps are placed proximal and distal to the site of planned cannulation, and a longitudinal arteriotomy is made. An $8 \mathrm{~mm}$ Dacron graft is sewn to the arteriotomy in an endto-side fashion using 5-0 or 6-0 polypropylene suture. The graft may then be cannulated, with the canula secured in place using multiple silk ties. Clamps are removed from the femoral artery and the canula de-aired in the standard fashion. At case conclusion the side-arm graft may be stapled or oversewn near its attachment to the native artery, with any residual graft material amputated.

\section{Axillary cannulation}

Axillary artery perfusion may be useful as it is less often compromised by significant occlusive disease compared to the femoral vessels, and provides antegrade arterial perfusion to reduce risk of stroke in patients with significant aortic atheromatous disease. The risk of limb ischemia may also be reduced compared to femoral cannulation due to significant arterial collateralization around the shoulder. A 4-5 cm infraclavicular incision is made approximately $1 \mathrm{~cm}$ beneath the lateral third of the clavicle. Fibers of the pectoralis major and minor may be divided or split. Care must be exercised during dissection to prevent any brachial plexus injury or entrapment during arterial control. After exposing a $2-3 \mathrm{~cm}$ arterial segment, and with the patient fully heparinized, the proximal and distal axillary artery are controlled with elastic vessel loops. A longitudinal arteriotomy is created and an $8 \mathrm{~mm}$ Dacron graft sewn to the artery in an end-to-side fashion, using 5-0 or 6-0 polypropylene suture. The side-arm graft may now be cannulated, secured, and de-aired in similar fashion to the femoral side-arm graft technique. At case conclusion the 
side-arm graft may be similarly stapled or oversewn as described above.

\section{Percutaneous}

Percutaneous femoral cannulation for CPB represents the least invasive approach, and is safe in well-selected patients. Generally, non-obese patients with adequate caliber non-diseased iliofemoral vasculature may be candidates, based on clinical and radiographic assessment, as well as surgeon comfort level. Non-obese habitus not only aids ultrasound-guided access for cannulation, but also improves diagnostic utility of the postoperative physical exam and any ultrasound studies when seeking to rule out access site complications such as pseudoaneurysm, hematoma with femoral nerve compression, or dissection.

\section{Arterial cannulation}

We begin with ultrasound assessment of the groin, taking care to identify the CFA, and its superficial and profunda branches to ensure appropriate access to the CFA using the Seldinger technique. The patient is heparinized after safe wire access has been confirmed. After skin and soft tissue dilation, a wire is advanced under TEE guidance into the descending aorta, and the CFA is dilated to at least $8 \mathrm{Fr}$ to accommodate preemptive deployment of the Proglide ${ }^{\mathrm{TM}}$ vascular closure device. After two vascular closure devices have been deployed, again under TEE visualization of the descending aorta, the CFA may be further dilated to accommodate the appropriately sized arterial cannula, which is then secured in place. After completion of the operation and weaning of $\mathrm{CPB}$, the arterial cannula may be directly accessed with needle puncture to allow resumption of wire access to the descending aorta. While the surgeon keeps gentle tension on the previously placed Proglide ${ }^{\mathrm{TM}}$ sutures, the assistant removes the arterial cannula while keeping forward pressure on the guidewire to maintain arterial wire access. After the Proglide ${ }^{\mathrm{TM}}$ knots are advanced to the level of the arteriotomy, and if hemostasis is adequate, the guidewire may be removed. The suture knots are then secured and cut. Gentle pressure is held for 10 minutes, and ipsilateral femoral and distal pulses are confirmed at case conclusion.

\section{Venous cannulation}

Similar to arterial access, the common femoral vein is positively identified and accessed under ultrasound guidance using the Seldinger technique. Guidewire advancement into the SVC is ensured by a bicaval view on TEE, and may be troubleshot as outlined above. After appropriate SVC guidewire access, we measure the venous cannula on the patient externally to anticipate appropriate depth of placement and place a purse-string suture around the site of skin entry to be secured at the time of decannulation. The venous cannula is slowly advanced under TEE guidance to an appropriate depth, and secured in place. At case conclusion and after $\mathrm{CPB}$ weaning the venous cannula may be slowly removed, the previously placed purse-string suture is tied, and gentle pressure held for approximately 5 minutes.

\section{Aortic occlusion}

Despite the need for creative techniques to achieve reliable aortic occlusion and myocardial protection in minimally invasive surgery, the goals of complete aortic occlusion and adequate de-airing for stroke prevention remain paramount, while seeking to prevent injury to the aorta and adjacent structures, and minimize encroachment on the operative field.

\section{Aortic cross clamping}

Aortic occlusion by cross clamping can be achieved directly through the operative field, or utilizing a separate chest incision. This technique generally proves inexpensive, as the clamps are reusable, in comparison to the endoballoon technique. We prefer using a flexible cross clamp through our access incision during direct-access minimally invasive mitral cases. Alternatively, a straight Chitwood clamp can be placed through a separate anterior $5 \mathrm{~mm}$ skin incision in the $2^{\text {nd }}$ or $3^{\text {rd }}$ intercostal space, taking care to avoid SVC compression, or injury to the pulmonary artery and left atrial appendage. Transthoracic aortic cross clamping obviously requires an additional aortic cannulation site for antegrade cardioplegia delivery, either exclusively or in combination with retrograde cardioplegia. We typically deliver antegrade Del Nido cardioplegia through a cardioplegia needle placed directly through our operative field.

\section{Endoaortic balloon}

An alternative to direct aortic cross clamping and requirement of a separate aortic puncture for cardioplegia 
delivery is to use an endoaortic balloon for both aortic occlusion and antegrade cardioplegia delivery. The endoaortic balloon is typically delivered via the femoral arterial canula, but if significant flow limitations and elevated arterial line pressures are created, it may be delivered through the contralateral femoral artery as well. TEE guidance is used to position a guidewire in the ascending aorta, over which the endoaortic balloon catheter is advanced. When the catheter tip is confirmed to be just above the sinotubular junction, the guidewire may be removed and the central lumen of the balloon catheter de-aired. Balloon and aortic root pressure transducers are confirmed to be working prior to balloon inflation. Saline inflation of the balloon is commenced incrementally, under TEE visualization, to reach a pressure goal of 350-450 mmHg. TEE guidance throughout the procedure is key to avoid aortic dissection or injury during balloon insertion, positioning, and deployment, and to assess for balloon migration. After balloon deployment, endoaortic cardioplegia may be delivered antegrade directly into the aortic root.

Controversy remains regarding the safety profiles of endoaortic balloon occlusion versus aortic clamping. While a previous large institutional series did not demonstrate significant differences in dissection or stroke (10), a more recent meta-analysis demonstrated a significantly greater rate of aortic dissection when an endoballoon, compared to a transthoracic clamp, was used for aortic occlusion during minimally invasive mitral surgery (11). In the same metaanalysis, rates of stroke, acute kidney injury, and all-cause mortality were similar between groups.

\section{Fibrillatory arrest}

This approach to myocardial protection has limited utility for minimally invasive mitral surgery and is not used by our team. Any significant degree of aortic regurgitation, whether de novo or secondary to left atrial retraction, may obscure the operative field with blood. The ability to de-air the left heart for stroke prevention is significantly limited, even with $\mathrm{CO}_{2}$ insufflation throughout the operative field. A previous examination of over 4,000 patients undergoing minimally invasive mitral surgery from the STS database demonstrated approximately threefold increased stroke risk when comparing fibrillatory arrest to aortic cross-clamping (5).

\section{Comments}

Minimally invasive mitral surgery includes a variety of operative exposures and options for cannulation and the conduct of CPB. By illustrating our technique, avoidable pitfalls, and alternatives, we hope to increase exposure to relevant nuances involved in safe conduct of minimally invasive mitral operations.

\section{Acknowledgments}

Funding: None.

\section{Footnote}

Provenance and Peer Review: This article was commissioned by the Guest Editor (Peyman Sardari Nia) for the series "Minimally Invasive Mitral Valve Surgery" published in Journal of Visualized Surgery. The article has undergone external peer review.

Conflicts of Interest: The series "Minimally Invasive Mitral Valve Surgery" was commissioned by the editorial office without any funding or sponsorship. TCN is Consultant for Edwards LifeSciences, Abbott, and LivaNova. The authors have no other conflicts of interest to declare.

Ethical Statement: The authors are accountable for all aspects of the work in ensuring that questions related to the accuracy or integrity of any part of the work are appropriately investigated and resolved. All procedures performed in studies involving human participants were in accordance with the ethical standards of the institutional and/or national research committee(s) and with the Helsinki Declaration (as revised in 2013). Written informed consent was obtained from the patient for publication of this manuscript and any accompanying images.

Open Access Statement: This is an Open Access article distributed in accordance with the Creative Commons Attribution-NonCommercial-NoDerivs 4.0 International License (CC BY-NC-ND 4.0), which permits the noncommercial replication and distribution of the article with the strict proviso that no changes or edits are made and the original work is properly cited (including links to both the formal publication through the relevant DOI and the license). See: https://creativecommons.org/licenses/by-nc-nd/4.0/.

\section{References}

1. Chitwood WR Jr, Elbeery JR, Chapman WH, et al. 
Video-assisted minimally invasive mitral valve surgery: the "micro-mitral" operation. J Thorac Cardiovasc Surg 1997;113:413-4.

2. Chitwood WR Jr, Wixon CL, Elbeery JR, et al. Videoassisted minimally invasive mitral valve surgery. J Thorac Cardiovasc Surg 1997;114:773-80; discussion 780-2.

3. Cohn LH, Adams DH, Couper GS, et al. Minimally invasive cardiac valve surgery improves patient satisfaction while reducing costs of cardiac valve replacement and repair. Ann Surg 1997;226:421-6; discussion 427-8.

4. Cosgrove DM 3rd, Sabik JF, Navia JL. Minimally invasive valve operations. Ann Thorac Surg 1998;65:1535-8; discussion 1538-9.

5. Gammie JS, Zhao Y, Peterson ED, et al. Maxwell Chamberlain Memorial Paper for adult cardiac surgery. Less-invasive mitral valve operations: trends and outcomes from the Society of Thoracic Surgeons Adult Cardiac Surgery Database. Ann Thorac Surg 2010;90:1401-8, 10.e1; discussion 1408-10.

6. Youssef SJ, Millan JA, Youssef GM, et al. The role of computed tomography angiography in patients undergoing evaluation for minimally invasive cardiac surgery: an early program experience. Innovations (Phila) 2015;10:33-8.

doi: 10.21037 /jovs.2018.12.10

Cite this article as: Nissen AP, Abreu J, Ornekian V, Nguyen S, Nguyen TC. Percutaneous and open cannulation for peripheral cardiopulmonary bypass: pearls and pitfalls. J Vis Surg 2018;4:256.
7. Ailawadi G, Agnihotri AK, Mehall JR, et al. Minimally Invasive Mitral Valve Surgery I: Patient Selection, Evaluation, and Planning. Innovations (Phila) 2016;11:243-50.

8. Nissen AP, Abreu J, Ornekian V, et al. Our preferred techniques for both direct and percutaneous femoral cannulation in minimally invasive mitral surgery. Asvide 2018;5:930. Available online: http://www.asvide.com/ article/view/29187

9. Tarui T, Miyata K, Shigematsu S, et al. Risk factors to predict leg ischemia in patients undergoing single femoral artery cannulation in minimally invasive cardiac surgery. Perfusion 2018;33:533-7.

10. Atluri P, Goldstone AB, Fox J, et al. Port access cardiac operations can be safely performed with either endoaortic balloon or Chitwood clamp. Ann Thorac Surg 2014;98:1579-83; discussion 1583-4.

11. Kowalewski M, Malvindi PG, Suwalski P, et al. Clinical Safety and Effectiveness of Endoaortic as Compared to Transthoracic Clamp for Small Thoracotomy Mitral Valve Surgery: Meta-Analysis of Observational Studies. Ann Thorac Surg 2017;103:676-86. 